\title{
Correction to: Acacia hydaspica R. Parker ameliorates cisplatin induced oxidative stress, DNA damage and morphological alterations in rat pulmonary tissue
}

Tayyaba Afsar ${ }^{1}$, Suhail Razak ${ }^{2,3^{*}}$, Ali Almajwal ${ }^{3}$ and Muhammad Rashid Khan ${ }^{1}$

\section{Correction to: BMC Complement Altern Med (2018) 18:49}

https://doi.org/10.1186/s12906-018-2113-0

Following publication of the original article [1], the author reported that Tables 3 and 4 were incorrect due to a production error.

The corrected tables are given below.

\section{Author details}

'Department of Biochemistry, Faculty of Biological Sciences, Quaid-i-Azam University, Islamabad, Pakistan. ${ }^{2}$ Department of Animal Sciences, Faculty of Biological Sciences, Quaid-i-Azam University, Islamabad, Pakistan.

${ }^{3}$ Department of Community Health Sciences, College of Applied Medical

Sciences, King Saud University, Riyadh, Saudi Arabia.

Published online: 07 November 2019

\section{Reference}

1. Afsar, et al. Acacia hydaspica R. Parker ameliorates cisplatin induced oxidative stress, DNA damage and morphological alterations in rat pulmonary tissue. BMC Complement Altern Med. 2018;18:49. https://doi.org/ 10.1186/s12906-018-2113-0

* Correspondence: ruhail12345@yahoo.com

2Department of Animal Sciences, Faculty of Biological Sciences, Quaid-i-Azam University, Islamabad, Pakistan

${ }^{3}$ Department of Community Health Sciences, College of Applied Medical

Sciences, King Saud University, Riyadh, Saudi Arabia

Full list of author information is available at the end of the article

(c) The Author(s). 2019 Open Access This article is distributed under the terms of the Creative Commons Attribution 4.0 International License (http://creativecommons.org/licenses/by/4.0/), which permits unrestricted use, distribution, and reproduction in any medium, provided you give appropriate credit to the original author(s) and the source, provide a link to the Creative Commons license, and indicate if changes were made. The Creative Commons Public Domain Dedication waiver (http://creativecommons.org/publicdomain/zero/1.0/) applies to the data made available in this article, unless otherwise stated. 
Table 3 Effect of cisplatin (CP) and different treatments of AHE on lung tissue antioxidant enzymes

\begin{tabular}{lllll}
\hline Group & $\begin{array}{l}\text { POD } \\
(\mathrm{U} / \mathrm{min})\end{array}$ & $\begin{array}{l}\text { SOD } \\
(\mathrm{U} / \mathrm{mg} \text { protein })\end{array}$ & $\begin{array}{l}\text { CAT } \\
(\mathrm{U} / \mathrm{min})\end{array}$ & $\begin{array}{l}\text { QR } \\
(\mathrm{nM} / \mathrm{min} / \mathrm{mg} \mathrm{protein})\end{array}$ \\
\hline Control & $9.56 \pm 0.635^{\mathrm{b}}$ & $1.366 \pm 0.038^{\mathrm{b}}$ & $18.48 \pm 0.058^{\mathrm{b}}$ & $128.2 \pm 0.81^{\mathrm{b}}$ \\
CP & $5.03 \pm 0.271^{\mathrm{a}}$ & $0.765 \pm 0.019^{\mathrm{a}}$ & $10.83 \pm 0.049^{\mathrm{a}}$ & $83.99 \pm 0.486^{\mathrm{a}}$ \\
AHE alone & $10.29 \pm 0.314^{\mathrm{b}}$ & $1.358 \pm 0.058^{\mathrm{b}}$ & $18.57 \pm 0.057^{\mathrm{b}}$ & $128.9 \pm 0.179^{\mathrm{b}}$ \\
CP + AHE & $6.22 \pm 0.128^{\mathrm{a}, \mathrm{d}}$ & $0.982 \pm 0.035^{\mathrm{a}, \mathrm{b}^{*}, \mathrm{~d}^{* *}}$ & $14.15 \pm 0.083^{\mathrm{a}, \mathrm{b}, \mathrm{d}}$ & $98.82 \pm 1.232^{\mathrm{a}, \mathrm{b}, \mathrm{d}}$ \\
AHE + CP & $9.18 \pm 0.185^{\mathrm{b}, \mathrm{c}}$ & $1.255 \pm 0.038^{\mathrm{b}, \mathrm{c}^{* *}}$ & $17.07 \pm 0.026^{\mathrm{a}, \mathrm{b}, \mathrm{c}}$ & $119.5 \pm 1.283^{\mathrm{a}, \mathrm{b}, \mathrm{c}}$ \\
$\mathrm{CP}+$ Sily & $9.20 \pm 0.208^{\mathrm{b}}$ & $1.262 \pm 0.021^{\mathrm{b}}$ & $17.14 \pm 0.081^{\mathrm{a}, \mathrm{b}}$ & $119.9 \pm 1.008^{\mathrm{a}, \mathrm{b}}$ \\
\hline
\end{tabular}

Values expressed as mean \pm SEM. ${ }^{\text {a }}$ Significance at $p<0.0001$ Vs. control group, ${ }^{\text {b }}$ Significance at $p<0.0001$ Vs. Cisplatin (CP) group. ${ }^{\mathrm{c}}$ Significance at $p<0.0001$ of $\mathrm{AHE}+\mathrm{CP}$ pre-treated group Vs. CP + AHE post-treated group. ${ }^{\mathrm{d}}$ Significance at $p<0.0001$ of CP + AHE treatment groups Vs CP + Sily group. ${ }^{*}$, ${ }^{* *}:$ Significant difference at $p<0.001$. Non-significant difference $(p>0.05)$ was recorded between control and AHE alone treated group in all parameters (One way ANOVA followed by Tukey's multiple comparison tests)

Table 4 Effect of cisplatin (CP) and different treatments of AHE on lungs tissue antioxidant enzymes and GSH profile

\begin{tabular}{|c|c|c|c|c|c|}
\hline Group & $\begin{array}{l}\text { GSH } \\
(\mu \mathrm{M} / \mathrm{g} \text { tissue })\end{array}$ & $\begin{array}{l}\text { GR } \\
\text { (nM/min/mg protein) }\end{array}$ & $\begin{array}{l}\text { GST } \\
\text { (nM/min/mg protein) }\end{array}$ & $\begin{array}{l}\text { Y-GT } \\
\text { (nM/min/mg Protein) }\end{array}$ & $\begin{array}{l}\text { GPx } \\
\text { (nM/min/mg Protein) }\end{array}$ \\
\hline Control & $16.12 \pm 0.578^{b}$ & $143.7 \pm 1.342^{b}$ & $98.85 \pm 0.918^{b}$ & $295.4 \pm 1.113^{b}$ & $107.4 \pm 0.730^{b}$ \\
\hline $\mathrm{CP}$ & $8.334 \pm 0.356^{a}$ & $98.02 \pm 0.619^{a}$ & $68.17 \pm 0.962^{a}$ & $82.82 \pm 0.958^{a}$ & $54.08 \pm 0.909^{a}$ \\
\hline AHE alone & $6.38 \pm 0.207^{b}$ & $144.0 \pm 1.492^{b}$ & $99.79 \pm 1.865^{b}$ & $295.6 \pm 0.599^{b}$ & $108.8 \pm 1.216^{\mathrm{b}}$ \\
\hline $\mathrm{CP}+\mathrm{AHE}$ & $11.99 \pm 0.305^{a, b, d}$ & $116.9 \pm 0.813^{a, b, d}$ & $78.34 \pm 1.076^{a, b^{* *}, d^{* *}}$ & $137.8 \pm 1.017^{a, b, d}$ & $71.28 .8 \pm 0.501^{a, b, c}$ \\
\hline $\mathrm{AHE}+\mathrm{CP}$ & $15.63 \pm 0.532^{b, c}$ & $135.0 \pm 0.393^{a, b, c}$ & $89.65 \pm 1.49^{a * *, b, c}$ & $261.4 \pm 0.802^{a, b, c}$ & $92.78 \pm 1.216^{a, b, c}$ \\
\hline $\mathrm{CP}+$ Sily & $15.29 \pm 0.312^{b}$ & $133.8 \pm 1.25^{a, b}$ & $87.60 \pm 1.644^{a, b}$ & $264.3 \pm 1.067^{\mathrm{a}, \mathrm{b}}$ & $95.64 \pm 1.573^{\mathrm{a}, \mathrm{b}}$ \\
\hline
\end{tabular}

Values expressed as mean \pm SEM. ${ }^{\text {a }}$ Significance at $p<0.0001$ Vs. control group, ${ }^{\mathrm{b}}$ Significance at $p<0.0001 \mathrm{Vs}$. Cisplatin (CP) group. ${ }^{\mathrm{C}}$ Significance at $p<0.0001$ of $\mathrm{AHE}+\mathrm{CP}$ pre-treated group Vs. CP + AHE post-treated group. ${ }^{\mathrm{d}}$ Significance at $p<0.0001$ of CP + AHE treatment groups Vs CP + Sily group. ${ }^{*},{ }^{* *}:$ Significant difference at $p<0.001$. Non-significant difference $(p>0.05)$ was recorded between control and AHE alone treated group in all parameters (One way ANOVA followed by Tukey's multiple comparison tests) 